\title{
Antoni M. Alcover, le narrateur généreux
}

\author{
Jaume Guiscafrè
}

\section{(2) OpenEdition}

Journals

Édition électronique

URL : https://journals.openedition.org/clo/1687

DOI : $10.4000 /$ clo. 1687

ISSN : 2266-1816

Éditeur

INALCO

\section{Édition imprimée}

Date de publication : 2 janvier 2012

ISBN : 978-2-85831-212-2

ISSN : 0396-891X

\section{Référence électronique}

Jaume Guiscafrè, «Antoni M. Alcover, le narrateur généreux », Cahiers de littérature orale [En ligne], 72 |

2012, mis en ligne le 29 avril 2015, consulté le 01 juillet 2021. URL : http://journals.openedition.org/ clo/1687 ; DOI : https://doi.org/10.4000/clo.1687

Ce document a été généré automatiquement le 1 juillet 2021.

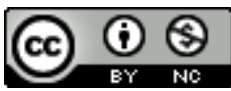

Cahiers de littérature orale est mis à disposition selon les termes de la Licence Creative Commons Attribution - Pas d'Utilisation Commerciale 4.0 International. 


\title{
Antoni M. Alcover, le narrateur généreux
}

\author{
Jaume Guiscafrè
}

1 Antoni Maria Alcover Sureda (Manacor, 1862 - Palma, 1932) est une des personnalités les plus remarquables de la culture catalane : par son caractère entêté et indomptable, par sa ténacité et sa capacité de travail et, surtout, par l'importance et l'impact de son œuvre lexicographique et littéraire ${ }^{1}$. Dans cet article, je me concentrerai uniquement sur un aspect de sa production que lui-même considérait comme marginal, à savoir son œuvre de collecteur et de narrateur de contes de tradition orale ${ }^{2}$.

2 Pour comprendre et expliquer les particularités de cette partie de la production littéraire d'Alcover, il convient de ne pas oublier ce qui a initialement éveillé son intérêt pour le conte oral : le fait qu'il se soit spécialisé dans la rondallística - si l'on peut dire, au sens strict, qu'il s'est consacré à la rondallística, c'est-à-dire à l'étude des contes majorquins - fut une conséquence de son goût pour la lecture :

Aux alentours de 1879 [...] sont tombés entre mes mains les livres de l'écrivain populaire espagnol Antonio de Trueba, qui furent pour moi une véritable révélation car l'espagnol tel que le parlaient les personnages des livres de Trueba m'était inconnu, beaucoup de ses rondalles étaient racontées dans le même style que celles que j'avais entendu raconter par mon père, ma mère et les gens qui habitaient autour de chez nous, qui les racontaient, je suppose, en majorquin, et que Trueba racontait dans un langage très différent de celui des livres en espagnol que j'avais lus jusqu'alors.

(Alcover, 1983, 123).

3 Je tiens à signaler deux aspects qui ont retenu à dix-sept ans l'attention du jeune Alcover et qui expliquent en quelque sorte la singularité de sa production narrative postérieure. D'une part les contes de Trueba ${ }^{3}$ étaient du style de ceux qui lui étaient familiers: Alcover [re]découvre donc sa propre tradition narrative - celle de son entourage, d'abord - à la lecture de l'œuvre de Trueba et non pas comme conséquence d'un intérêt spécial ou d'une intention particulière, encore moins d'une réflexion théorique préalable sur la narration orale ou le folklore; en fin de compte, lui-même participait intégralement de la tradition à laquelle il commençait à s'intéresser ainsi 
qu'à la dynamique de transmission du conte oral. D'autre part, les récits de l'auteur basque étaient racontés dans un "langage très différent de celui employé dans les livres ", ce qui lui permet de découvrir qu'il existe un type de littérature qui peut être élaboré dans un langage autre que « livresque » ou « littéraire ».

Suite à cet élément déclencheur initial, Alcover va donc commencer « à essayer d'écrire en espagnol, en imitant le langage de Trueba, des contes majorquins comme il les avait entendus raconter ». Mais c'est alors qu'il se trouve face à un sentiment d'étrangeté dû au fait qu'il racontait en espagnol des histoires qu'il n'avait entendues jusque-là qu'en catalan. Josep Miralles, un camarade de séminaire, plus tard évêque de Majorque, lui fit comprendre que ces choses-là, il «ne devait pas les écrire en espagnol populaire ni littéraire mais en majorquin dépouillé, tel que le parlent les majorquins qui n'ont pas fait d'études " et malgré sa réticence initiale, convaincu que ce que lui proposait Miralles était «une idée saugrenue et antilittéraire» (Alcover, 1983,123), Alcover parvint finalement à s'en convaincre et abandonna très vite la langue espagnole en tant qu'outil de création littéraire. En fait, c'était la seule solution possible et admissible si son intention était, comme nous le verrons, de reprendre des versions de la tradition orale, car toute autre solution aurait fait obstacle au passage à l'écrit de la littérature orale majorquine ou en aurait limité la vraisemblance en altérant une de ses caractéristiques fondamentales : le dialectalisme radical.

C'est ainsi qu'il va publier en 1880, dans le numéro 76 de la revue L'Ignorància, le premier échantillon de sa production de contes Es jai de sa barraqueta ( Le vieux de la cabane »), une version d'ATU 330, et neuf ans plus tard, durant l'été 1889, il eut l'idée "de faire un livre composé uniquement de contes populaires vivants de Majorque ", une idée qui n'allait se matérialiser qu'à la fin de l'année 1896 avec la publication du premier volume de l'Aplec de Rondaies Mallorquines d'En Jordi d'es Racó (Recueil de contes majorquins de Jordi d'es Racó). L'activité de collecteur et d'éditeur d'Alcover durera environ cinquante et un ans durant lesquels il recueillera, de façon intermittente, des versions orales et il en publiera les réélaborations aussi bien dans la presse périodique que sous forme de volume. Toutefois, celle-ci n'était pas, loin de là, son activité prioritaire. Il reconnaissait lui-même qu'il s'adonnait à la collecte de ces versions et à leur réélaboration littéraire durant le temps que lui laissaient ses autres tâches et obligations, qui étaient effectivement nombreuses : il fut vicaire général, chanoine magistral, vicaire capitulaire et doyen dans la cathédrale de Palma ; agrégé d'Histoire de Majorque, d'Histoire Ecclésiastique et des Lieux Théologiques dans le scolasticat de Palma; philologue, linguiste, éditeur, historien, archéologue et même architecte. En 1928, il fut victime d'une première attaque d'apoplexie, ce qui allait le contraindre à réduire son temps de travail et à s'abstenir de tout travail intellectuel intense, circonstance dont il profitera pour s'attaquer à la rédaction définitive des versions des récits dont il n'avait que des notes et qui allaient constituer les volumes onze et douze, les deux derniers de la collection. À la mort d'Alcover, en 1932, son disciple et continuateur de son œuvre, Francesc de Borja Moll, reprit la publication des contes : il procéda à la restructuration des volumes et récupéra également, pour en faire de nouveaux volumes, des contes qui n'avaient paru que dans les revues ou qu'Alcover n'avait pas publiés. C'est en 1936 qu'il fit paraître le premier volume de ce qu'on appela «l'édition définitive » qui, en 1975, allait être composée de vingt-quatre volumes encore édités aujourd'hui ${ }^{4}$. 
6 Bien qu'il ait consacré une grande partie de son temps, quoique de façon intermittente, à la collecte et à la publication des contes oraux, Alcover n'a guère théorisé sur le genre, surtout du fait que - et ceci est un aspect essentiel de sa démarche - il s'est toujours comporté comme un rondallaire plutôt que comme un rondallista ${ }^{5}$. En catalan, cette distinction a son importance: rondallaire signifie "conteur de rondalles", par contre, un rondallista est une "personne qui étudie les rondalles, versée dans les rondalles, qui collecte des rondalles ». En ce sens, Alcover s'est plus consacré à rassembler et à réécrire les versions des contes qui allaient constituer l'Aplec qu'à réfléchir sur la nature, l'origine, la diffusion ou la fonction de ce type de contes. C'est pourquoi, si nous le qualifions de « folkloriste ", un terme qui au sens strict n'est peutêtre pas très approprié, c'est par commodité et, en particulier, parce qu'il a réalisé un certain travail de terrain avant la rédaction et la publication de ses propres versions et a indiqué ses sources d'information. Finalement, toute la théorisation d'Alcover sur le conte oral est contenue dans trois textes : les prologues aux deux premières éditions du premier volume de l'Aplec de Rondaies Mallorquines, publiées en 1896 et 1915, et « Com he fet mon Aplech de Rondaies Mallorquines», («Comment j’ai fait mon Recueil de Contes majorquins »), article qui, à la demande de Leo Spitzer, fut publié en 1931 au volume 51 de la Zeitschrift für romanische Philologie et qui n'est autre qu'une version refondue et élargie des deux prologues ${ }^{6}$.

7 Dans le prologue du premier volume, Alcover explique déjà très clairement la finalité de la publication de ses propres versions et le critère appliqué dans la constitution de cette collection de textes qui allaient devenir l'Aplec de Rondaies Mallorquines :

Je n'ai pas fait ce recueil dans un but technique ni scientifique mais plutôt comme un hommage d'affection filiale pour notre chère langue et notre tradition vénérée, et je le destine au peuple majorquin et à la propagande de la sainte cause qui a pour devise Dieu et Patrie.

8 Dans le prologue de la deuxième édition de ce même volume, il insiste à nouveau sur ce sujet en répétant presque mot à mot le texte ci-dessus mais en reformulant toutefois de façon significative la dédicace :

Je n'ai pas fait ce recueil dans un but technique ni scientifique mais plutôt comme un hommage d'affection filiale pour notre chère langue et notre tradition vénérée, et je l'ai dédié au peuple catalan, de Majorque et d'ailleurs.

Et il reprend encore cette même déclaration et la reformule pour la troisième fois :

Comment j'ai fait mon Recueil... : "Je ne l'ai pas fait dans un but technique ni exclusivement scientifique, mais dans un but patriotique. Je n'ai pas voulu faire un ouvrage pour les folkloristes, mais pour le peuple majorquin. »

10 J'ai vraiment l'impression que les reformulations successives de ces quelques lignes répondent à l'évolution des idées aussi bien « politiques » que « théoriques » d'Alcover. Les premières expliqueraient, par exemple, cette fluctuation dans la dédicace entre «peuple majorquin » et "peuple catalan ». Mais il est plus important, dans le cadre de ce travail, que nous nous concentrions sur les secondes, à propos d'une question qui a fait débat dès la publication des premiers volumes de la collection. Je me réfère à la valeur que les versions d'Alcover peuvent avoir en tant que document folklorique et, par conséquent, à l'utilité "scientifique» que peut avoir la collection pour les folkloristes qui suivront ${ }^{7}$. Je n'approfondirai pas cette question mais je tiens à souligner l'évolution de la vision qu'il avait de l'intérêt scientifique de ses propres textes. Dans les deux premières formulations, Alcover reconnaît qu'il n'a aucune prétention scientifique. Par contre, l'utilisation qu'il fait de l'adverbe exclusivement dans la 
dernière formulation laisse effectivement entendre qu'il y aurait eu également un but scientifique dans la collecte, la réélaboration et la publication de ces versions. Mais il y a encore un autre détail qui doit retenir notre attention, il s'agit de l'opposition entre le terme folkloristes, qui n'apparaît dans aucun des deux prologues, et le syntagme peuple majorquin auquel il adhère de façon assez explicite. Ces nuances pourraient sembler insignifiantes mais elles mettent en évidence le fait qu'au fur et à mesure que les études folkloriques progressaient et évoluaient, Alcover se montrait plus ou moins réceptif à ces changements ${ }^{8}$.

11 Toutefois cet intérêt n'aura en fin de compte aucune conséquence sur la publication des volumes de contes. D'une part, parce que l'objectif de l'auteur de Manacor n'a jamais été de se poser véritablement en folkloriste et, par conséquent, il n'a jamais atteint la condition d'outsider au sens que Lord $(1995,222)$ donne à ce terme: "those who are not members of the traditional group, who, therefore, approach the text without the assumptions and sense of values that the members of the traditional group share with one another" et, tout bien considéré, il n'aurait jamais pu y parvenir, puisque son activité de collecteur de versions orales s'est pratiquement limitée à son entourage et aux gens qu'il fréquentait régulièrement, du moins durant son enfance et son adolescence : les travailleurs de l'exploitation agricole où il était né, tous originaires de villages situés à l'est de Majorque 9 . D'autre part, parce que l'objectif final d'Alcover n'était pas seulement de témoigner d'une disparition et d'essayer de recueillir des œuvres de littérature orale dans un but archivistique avant que ce ne soit plus possible, mais aussi d'introduire des histoires et les narrations qui leur étaient associées dans des circuits et des dynamiques de communication dont elles étaient encore totalement exclues, du moins à Majorque : ceux de la littérature imprimée et de la lecture.

Toutefois, le passage de l'oral à l'écrit comporte un certain nombre de contraintes et un prix à payer inévitables qui, dans une plus ou moins grande mesure, dénaturent le récit oral. Lorsque la personne chargée de la transposition intersémiotique est, à proprement parler, un transcripteur, le texte qui en résulte peut conserver quelques traces de l'oralité dont il est issu. Mais ces traces peuvent néanmoins disparaître complètement si celui qui fait passer à l'écrit un récit oral se pose comme conteur. Alcover a naturellement été confronté à ces contraintes et à ce prix à payer lorsqu'il envisagea la rédaction des versions écrites des récits qui lui avaient été transmis par les conteurs oraux et qu'il avait consignés, de façon plus ou moins schématique, dans les six cahiers utilisés à cet effet ${ }^{10}$. Et il choisit sans hésiter de le faire en tant que conteur et non pas en tant que simple transcripteur:

Quel est le critère, quel est le système que j'ai appliqué pour écrire les rondalles? Si je me suis limité à les transcrire mot à mot telles qu'elles m'ont été racontées, sans rien y ajouter de personnel ni en changer la forme? Non, je ne l'ai jamais fait; et plus ça allait, plus j'étais convaincu que cela aurait été un échec, et non des moindres, si je m'étais plié à en faire une transcription aussi servile.

(Alcover, 1983, 124).

13 En fait, dans ces quelques lignes, l'auteur pose à nouveau le problème qui commençait à apparaître à propos du conte catalan, du moins à partir de 1868, lorsque Jaume Collell publia Cantem massa i parlem poc (« Nous chantons trop et parlons peu »), un article dans lequel il exhorte les hommes de lettres catalans à écrire en prose parce que :

il n'y a pas assez de poésie pour constituer une littérature au sens large du mot, il est nécessaire d'écrire aussi en prose et sous différentes formes littéraires pour que 
la physionomie littéraire d'un peuple soit complète et bien marquée.

(Collell, 1968, 17-18).

Dans ce texte, Collell ne se limite pas seulement à rendre son diagnostic public, il énonce aussi une série de propositions thématiques qui, selon lui, mériteraient un traitement littéraire en prose, parmi lesquelles figurent les "traditions poétiques », les " faits légendaires ", " un grand nombre de contes charmants » et " coutumes et fêtes traditionnelles » (Collell, 1868, 17-18). L'idée sous-jacente à la proposition de Collell est que la narration orale en tant que genre ne possède pas une valeur artistique autonome et appréciable, si ce n'est en termes d'utilité pratique : fournir des thèmes et des motifs aux narrateurs " littéraires " afin qu'ils puissent construire à partir de là les récits qui contribueront à donner de la visibilité et du prestige à un système littéraire en langue catalane : langue qui vient de naître et qui revendique un statut de langue de culture, aussi apte que toute autre à la représentation littéraire ${ }^{11}$. La clé de voûte de cette argumentation est ce que nous pourrions appeler "le problème de la forme", qu'Alcover explique dans les termes suivants, dans un paragraphe aux résonances herdériennes évidentes :

Que signifie populaire? Quelle est la forme authentique ? Où est l'âme du peuple, la pensée, le cœur du peuple? À travers qui l'âme du peuple s'exprime-t-elle ? À travers celui qui est vif d'esprit ou bien celui qui est lent d'esprit ? À travers celui qui est communicatif et parle comme un moulin à paroles ou à travers celui qui est réservé et avare de mots? Que répondront à ce sujet les partisans de recueillir les rondalles en se pliant servilement à transcrire juste ce que le peuple raconte, sans rien ajouter ni rien enlever à ce que dit celui qui la raconte ? [...] Si de chacune des rondalles, il fallait transcrire et publier la version de chaque conteur, qui résisterait à la lecture ? Seul y résisterait un spécialiste du folklore [...]. Il est certain qu'il faut recueillir chaque rondalla telle qu'elle est racontée par chacun des conteurs, mais ensuite le collecteur doit tirer de toutes ces versions, différentes et discordantes, la forme qu'il considère la plus fidèle et la plus proche de la façon de sentir du peuple, la forme pouvant reproduire avec le plus d'exactitude le caractère, la façon de voir et de sentir les choses du peuple à travers lequel cette rondalla prend vie.

(Alcover, 1983, 124-125).

Il conviendrait peut-être de revenir maintenant à la distinction que j'ai faite plus haut entre rondallista et rondallaire, car les uns comme les autres durent résoudre ce problème. Aux folkloristes qui se comportaient comme des rondallistes, qui appréciaient la valeur esthétique de la façon de procéder des narrateurs et des versions orales qu'ils recueillaient, il manquait la technologie qui leur aurait permis de consigner de façon fidèle les textes de ces versions. C'est pourquoi ils se sont vus contraints de les décrire afin de les reconstituer ou de les réélaborer postérieurement: s'ils n'y réussissaient pas, il ne leur restait plus qu'à y renoncer. De ce point de vue, Marià Aguiló, un des maitres d'Alcover, est un cas paradigmatique. Son échec avoué en ce qui concerne la recherche d'un langage adéquat pour la reconstitution ou la réélaboration « littéraire » des versions qu'il avait recueillies ou qu'il avait fait recueillir, est ce qui rend sa collection de contes intéressante pour les archives folkloriques et ce qui l'exclut dans le même temps de l'histoire de la littérature catalane. Cet échec a permis qu'un grand nombre de ces versions soient conservées dans un état de rédaction très proche des textes oraux dont elles sont issues, à l'instar de ce qui se passe dans le cas des notes $d^{\prime}$ Alcover ${ }^{12}$. Par contre, les folkloristes qui se comportaient comme des rondallaires, qui avaient une orientation essentiellement littéraire, se sentaient obligés de donner forme au matériel qu'ils recueillaient ou desquels ils s'inspiraient. Et pour y arriver, ils appliquaient de façon consciente toute une série de techniques de représentation et de 
procédés littéraires à la transcription des versions notées. Je parle d'application « consciente " par rapport à la remarque faite par Jason (1977, 6-7) à savoir que les narrateurs/interprètes oraux acquièrent et manient le canon, "a set of rules of composition and a lexicon of contentual units", de façon inconsciente. Et c'est cette particularité qui fait qu'ils sont traditionnels parce que "both the canon and the resulting ethnopoetic work remain almost inflexible and have been so since the beginning of literacy". Dans ce sens, pour que le texte résultant puisse être considéré comme réussi, "définitif »-, il fallait qu'ils trouvent un équilibre entre la simplicité de l'expression supposée qui caractérise les manifestations littéraires du vulgus in populo et le « désir de faire briller la langue », comme le disait Francesc Maspons, ce qui est, en fin de compte, une des aspirations propres de l'écrivain.

Dans le cas d'Alcover, ce processus d'élaboration d'un texte définitif, clos et durable, commence au moment même où il recueille les récits oraux qui lui servent de base. Et il convient de souligner qu'il s'agit du premier auteur d'une collection de contes de ce type qui, dans la littérature catalane, reconnait de manière explicite les sources orales qui sont à l'origine des récits qu'il publie, même s'il est vrai que parfois l'information qu'il donne n'est pas aussi complète qu'il le laisse entendre ${ }^{13}$ :

[...] dès le début, j'ai cru [...] qu'il convenait et qu'il était nécessaire de rassembler tout ce qu'on me conterait et tel que l'on me le conterait et de le consigner avec grande fidélité et exactitude sur mes cahiers de notes, en notant même les phrases les plus caractéristiques et les mots spéciaux employés par le conteur, afin que tout ceci et en comparant toutes les versions que je trouverais de la même rondalla, me serve de base pour la rédaction définitive. [...] En consignant sur mes cahiers toutes les notes concernant les rondalles de ce qui se passait dans un village ou un autre - notes concernant l'argument, incidents et phrases de chaque conteur, en notant scrupuleusement le nom et la filiation de chacun - c'est ainsi que j'avais et j'ai la base pour écrire les rondalles [...] en les habillant d'un langage et d'une phraséologie purement majorquine que, je crois que personne n'en doutera, je connais suffisamment et je fais preuve de la retenue nécessaire pour éviter l'utilisation de mots ou de phrases qui ne sont pas en usage parmi les gens du peuple ici à Majorque, lesquels n'ont pas la folie des mots espagnols et s'efforcent de parler en bon majorquin.

(Alcover, 1983, 125).

Le nombre de personnes ayant contribué par l'apport d'une version à la constitution de l'Aplec s'élève à deux cent vingt-huit. Cependant, toutes ces personnes ne méritent d'être considérées comme « conteurs ». En fait, la plupart ne l'étaient certainement pas, c'est-à-dire qu'ils ne possédaient pas de répertoire de contes plus ou moins fixe et récurrent qu'ils actualisaient en performances réelles, mais étaient plutôt des " informateurs ", c'est-à-dire des personnes qui connaissaient telle ou telle version et qui, de façon occasionnelle, en rendaient compte au folkloriste dans une performance de transmission. Il est fort probable que ce fut le cas d'Antoni Alcover et de Catalina Sureda, les parents d'Alcover qui apparaissent comme source, lui, dans vingt-neuf versions et elle, dans vingt-cinq. Par contre, les deux autres personnes qui apparaissent le plus souvent - vingt-sept fois chacune - dans les notes relatives aux sources orales des récits, devaient être des conteurs. Il s'agit d'Antoni Garrit, de Sant Llorenç del Cardassar qui, au dire d'Alcover, " en sait beaucoup et les raconte à merveille ", et de Rafela Quelona (Son Servera, 1861-1947), « une femme humble, aveugle et pauvre » qui est sans doute " la plus emblématique et la plus admirable » des conteurs dont on ait connaissance (Ballester, 2009, 37). Cependant, je ne crois pas - et il n'y en a aucune évidence - que, même dans le cas des véritables conteurs, Alcover transcrivît jamais des 
versions directement données dans une performance, mais plutôt que la méthode d'obtention des récits fut, à quelques exceptions près, celle de l'entretien, une situation communicative dans laquelle on ne peut parler de «narrateur » et d' " auditoire » - du fait qu'il ne s'agit pas d'un acte de communication folklorique -, mais d'« informateur " et d'« enquêteur " ${ }^{14}$. Je viens de dire "à quelques exceptions près " parce qu'il y a des récits qu'Alcover réélabore à partir de sa propre mémoire, notamment ceux qu'il a entendus raconter dans son entourage familial lorsqu'il était enfant ou adolescent, ce qui explique qu'il s'y réfère à l'imparfait au lieu du passé simple et qu'il n'y en a aucune version dans ses cahiers de notes.

Mais dans cette reconnaissance explicite de ses sources, Alcover oscille entre son travail de folkloriste et sa veine de narrateur... et il privilégie une fois de plus cette dernière. Car même si, en tant que folkloriste, il apprécie les particularités narratives et linguistiques de chaque conteur et aspire même à les consigner par écrit et à en laisser un témoignage, en tant que narrateur, il s'en éloigne complètement et en fait disparaître toute trace ou vestige dans la rédaction définitive de ses versions : dans certains cas, parce qu'il refond diverses versions orales en une seule version écrite, dans d'autres cas, parce qu'il reconstruit des versions qui lui paraissent incomplètes ou défectueuses ${ }^{15}$ et, en définitive, parce qu'il superpose et impose toujours son style narratif au détriment de celui des informateurs, de telle façon que les particularités stylistiques de chacun s'estompent et se diluent. Au moment même où un rondallista recrée ou réélabore un texte de provenance orale, il abandonne son rôle de rondallista pour assumer celui de rondallaire, c'est-à-dire d'auteur. Alors, inévitablement, sa personnalité apparaît à travers le nouveau texte parce que la seule personnalité visible de l'écrivain - en tout cas, la seule qui soit accessible au lecteur - est représentée par le langage. C'est pour cela, parce que l'écrivain y a imposé son propre langage, que nous chercherions en vain, dans les contes de la collection d'Alcover, les variations stylistiques que nous pourrions trouver dans un recueil de versions enregistrées ou transcrites provenant de narrateurs différents.

19 La voix du narrateur alcovérien peut donc être définie principalement par quatre caractéristiques que je me limiterai ici à mentionner. Premièrement, par l'utilisation de l'expansion comme procédé de transformation des matériaux manuscrits contenus dans ses cahiers de notes, un procédé qui se caractérise surtout par "une sorte de dilatation stylistique »: «il s'agit ici de doubler ou de tripler la longueur de chaque phrase de l'hypotexte ", comme le dit Genette $(1982,372)$ de façon caricaturale, et c'est exactement ce que fait le narrateur alcovérien ${ }^{16}$. Deuxièmement, par l'équilibre auquel il parvient entre le style réaliste - «dans son état classique, l'expansion n'explore qu'une direction stylistique, celle que je qualifiais faute de mieux d' "animation réaliste" " (Genette, 1982,374) - et le style abstrait, surtout propre au conte merveilleux ; un équilibre que Grimalt $(2003,579)$ considère être un des secrets qui font d'Alcover "un maître des narrateurs ". Troisièmement, par le fait qu'il applique au conte merveilleux - prédominant dans l'Aplec - grâce à la manipulation artistique du langage et aux procédés littéraires utilisés, la cosmovision carnavalesque si bien définie et décrite par Bakhtine et, last but not least, par l'extraordinaire rendement créatif, sans égal à ce jour, qu'il est capable d'obtenir de l'usage du dialecte majorquin ${ }^{17}$.

Ainsi, à la différence d'Aguiló, Alcover est arrivé, lui - avec difficulté, il est vrai -, à trouver son propre modèle de langue littéraire sans jamais avoir eu besoin de faire aucun type de déclaration ou de témoignage de fidélité aux versions orales parce qu'il 
n'a jamais eu l'intention de constituer un catalogue de textes scientifiquement homologables pour la folkloristique, mais seulement - et ce n'est pas peu!- une collection de récits réussis du point de vue littéraire et susceptibles d'entrer à nouveau dans la dynamique de la transmission orale ou, du moins, d'y interagir.

21 Malgré les nombreuses différences séparant les versions imprimées des versions orales qui lui avaient été transmises, Alcover a amplement atteint l'objectif qu'il s'était fixé lors de leur publication:

J'ai fait mon recueil par amour des rondalles, pour les sauver du naufrage, de la disparition, de l'oubli qui les menaçait. Je les ai rassemblées, écrites et publiées pour qu'elles puissent circuler à nouveau parmi les majorquins [...] c'est pour ça que j'ai écrit les rondalles de façon à ce qu'elles puissent être lues en famille.

(Alcover, 1983, 126).

22 Et il y est très largement parvenu car non seulement les lecteurs et les auditeurs majorquins en ont adopté les versions et en ont fait un référent canonique, mais aussi les narrateurs et les rondallaires ${ }^{18}$. Il est difficile d'évaluer avec exactitude l'influence qu'a pu avoir le canon alcovérien sur les conteurs oraux majorquins parce que nous disposons de très peu de témoignages, mais à partir du peu que nous savons avec certitude, je dirais qu'elle a été importante, le contraire serait étonnant ${ }^{19}$. Par contre, l'impact de l'Aplec, lui, est bien perceptible chez les rondallaires qui publièrent, postérieurement à Alcover, des contes provenant de la tradition orale et il se concrétise en trois aspects: d'une part, ces auteurs choisissent habituellement de publier des textes correspondant à des types "inédits", dans le sens où il n'en figure aucune version dans l'Aplec; d'autre part, ils renoncent de façon explicite à appliquer un traitement scientifique aux versions orales qu'ils ont recueillies; et, finalement, ils manifestent, à la manière d'un hommage, leur volonté explicite de rivaliser avec le langage et le style d'Alcover et ils tombent dans la contradiction en rédigeant des récits de provenance orale, apparemment libres de l'influence d'Alcover, mais qui s'inspirent de sa méthode et de son style. Un exemple significatif est le cas d'Orpí: dans le deuxième paragraphe de l'" Introducció » à ses Rondalles mallorquines inèdites - un volume au titre explicite -, il remémore « la découverte enfantine » de l'Aplec d'Alcover et, deux pages plus haut, il explique la genèse de son livre : il avait eu « l'idée de rédiger un jour, en suivant les critères du prélat Alcover, une poignée de contes dont je me souvenais de quand j'étais petit et qui ne figuraient pas dans l'Aplec » $(1996,15)$.

Cependant, les versions réélaborées d'Alcover n'ont pas seulement bénéficié d'un jugement favorable et de l'acceptation des rondallaires et des narrateurs oraux - qui, d'une façon ou d'une autre, les ont réintroduits dans les circuits et dans les dynamiques propres à la transmission orale -, mais près de trente ans après la mort de l'auteur, au début des années soixante $\mathrm{du} \mathrm{xx}^{\mathrm{e}}$ siècle, elles ont également accédé au champ de l'oralité radiophonique ou, pour employer les termes d'Ong $(2012,11)$, de l'oralité seconde, celle qui est propre à la culture technologique actuelle "in which a new orality is sustained by telephone, radio, television, and other electronic devices that depend for their existence and functioning on writing and print", ou de l'«oralité mécaniquement médiatisée » de Zumthor $(1983,36)$, "différée dans le temps et/ou l'espace ». À la demande du prêtre Guillem Fiol, directeur de la Ràdio Popular Diocesana de Mallorca, Francesc de Borja Moll fut chargé de la diffusion radiophonique des versions alcovériennes et il se souvient en ces termes de quelle fut sa tâche dans ce projet :

Ma tâche, en tant que directeur de l'émission « Rondaia mallorquina » n'était pas facile. Il fallait commencer par rayer de divers exemplaires du tome des Rondaies les 
passages à supprimer du fait qu'ils allongeaient trop l'émission à cause des répétitions ou de leur peu d'intérêt du point de vue radiophonique. Je notais, sur ces mêmes tomes, dans la marge, les initiales des différents personnages [...]. Après quoi, chaque acteur devait lire, chacun séparément, les morceaux qu'ils avaient à réciter et moi, je contrôlais la diction de chacun d'entre eux, leur faisant remarquer les défauts de prononciation, d'intonation, etc., et en leur recommandant toujours la vertu la plus importante d'un interprète : le naturel.

(Moll, 2003, 472).

Selon Grimalt $(1996,23)$, les textes d'Alcover "préservent au maximum les traits d'oralité » et, en référence, précisément, à ces émissions, il argumente que «leur aptitude à être lus à haute voix » le démontre. Ces appréciations se doivent toutefois d'être nuancées, même si nous pouvons admettre qu'il existe certains traits caractéristiques généralement associés à l'oralité qui survivent dans ces textes - la répétition, par exemple. Lorsqu'un narrateur actualise un récit en performance, l'oralité est le cadre dans lequel a lieu l'énonciation et elle la conditionne complètement. Par contre, les traits d'oralité que nous pouvons détecter dans les récits d'Alcover correspondent plutôt à une illusion d'oralité, à une oralité fictive, discursive, intradiégétique. Le milieu est différent et, par conséquent, plutôt que d'oralité, nous devrions parler d'un effet d'oralité, auquel Alcover parvient moyennant l'inclusion dans la diégèse de scènes dialoguées - la redondance est voulue - extraordinairement bien construites. Et c'est grâce à cette disposition "scénique", avec laquelle Alcover construit beaucoup de passages de ses versions, que Moll n'a pas eu beaucoup de difficultés pour les adapter. Mais lorsqu'il a fallu diffuser ces récits par un autre moyen de communication oral - la radio dans ce cas -, distinct de celui dont ils provenaient en dernière instance, ils ont dû passer obligatoirement par un stade intermédiaire - par un processus de transmodalisation, en fait, parce que nous sommes très proches de la dramatisation - où le directeur a éliminé aussi bien les traces d'oralité qui n'étaient pas adaptées à la nouvelle technique de diffusion, comme les passages répétitifs, ou autres éléments discursifs qui n'étaient pas pertinents dans une émission de radio et qui empêchaient une élocution fluide comme, par exemple, les digressions descriptives ou énumératives du conteur. Les émissions, en tout cas, ont connu un véritable succès : "Surtout les premières années, quand peu de gens encore avaient la télévision et tout le monde écoutait la radio; la rondalla majorquine était l'émission la plus écoutée de toutes celles de Ràdio Popular de Mallorca. En été, dans les rues de Palma, lorsque toutes les fenêtres étaient ouvertes, nous pouvions écouter raconter les rondalles dans toutes les maisons, au cours de notre promenade d'un bout à l'autre de la ville " $\left(\right.$ Moll, 2003, 472) ${ }^{20}$.

Alcover, avec son Aplec de Rondaies Mallorquines, a restitué généreusement, à toute une communauté, le trésor narratif que quelques-uns de ses membres lui avaient transmis. Et l'attention que le public majorquin - aussi bien ceux qui savaient lire que les illettrés - lui a accordée est en rapport avec la munificence de ce narrateur exubérant et généreux et l'aptitude qu'il a démontrée dans ses contes à s'adapter à des milieux divers et changeants :

Un des mérites que personne ne pourra lui refuser est celui d'avoir obtenu une approbation populaire qu'aucune autre œuvre littéraire ne s'est jamais vue accorder parmi nous. Le public majorquin a reconnu ces rondalles comme quelque chose qui lui appartient même dans leur aspect verbal: Alcover employait un langage qu'aucun majorquin ne parle mais beaucoup de lecteurs incapables d'accéder aux livres écrits en catalan, n'avaient aucune difficulté à le comprendre 
ni aucun inconvénient à le reconnaître comme leur.

(Grimalt, 1996, 24).

Les lecteurs ont donc décidé que l'âme du peuple majorquin parlait à travers Antoni M. Alcover qui s'était non seulement « le moins [éloigné] des paroles des innombrables conteurs anonymes» comme le prescrit Benjamin $(1991,206)$, mais qui les avait magnifiées et a su les restituer sur le mode de l'offrande généreuse à tous ceux qui ont voulu l'écouter ou le lire. C'est pour cela qu'il mérite de figurer parmi les grands.

\section{BIBLIOGRAPHIE}

ALCOVER, Antoni Maria, 1983, Com he fet mon «Aplec de Rondaies Mallorquines», Randa, $\mathrm{n}^{\circ}$ 14, p. 121-136.

ALCOVER, Antoni Maria, 1996, Aplec de Rondaies Mallorquines d'En Jordi d'es Racó, Ed. a/s de Josep A. Grimalt avec la collaboration de Jaume Guiscafrè, Palma de Mallorca, Editorial Moll [6 vol.]. BALLESTER, Ramon (ed.), 2009, Les rondalles més serverines, Palma de Mallorca, Editorial Moll. BENJAMIN, Walter, 1991, Le narrateur, Écrits français, Paris, Gallimard, coll. « Bibliothèque des idées », p. 205-229, [1 ${ }^{\text {re }}$ éd., 1936].

BENJAMIN, Walter, 2011, Le conteur. Expérience et pauvreté, Paris, Payot et Rivages, coll. « Petite Bibliothèque Payot », p. 51-106. Traduit de l'allemand par Cédric Cohen Skalli. COLLELL, Jaume, 1968, Cantem massa i parlem poc, Lo Gay Saber, any 1, nº 3, p. 17-18.

GENETTE, Gérard, 1982, Palimpsestes : La littérature au second degré, Paris, Éditions du Seuil.

GRIMALT, Josep A., 1978, La catalogació de les rondalles de mossèn Alcover com a introducció a llur estudi, Randa, $\mathrm{n}^{\circ}$ 7, p. 5-30.

GRIMALT, Josep A., 1994, Les «Notes de rondaies» de mossèn Alcover, entre l'oralitat i l'escriptura, Revista d'Etnologia de Catalunya, $\mathrm{n}^{\circ} 4$, p. 58-67.

GRIMALT, Josep A., 1996, Introducció, in Antoni Maria Alcover, 1996, Aplec de Rondaies Mallorquines d'En Jordi d'es Racó, Ed. a/s de Josep A. Grimalt avec la collaboration de Jaume Guiscafrè, Palma de Mallorca, Editorial Moll [6 vol.], p. 7-26.

GRIMALT, Josep A., 1998, Les rondalles reconstruïdes de mossèn Alcover, in Estudis de llengua $i$ literatura en honor de Joan Veny, 2. Barcelona, Publicacions de l'Abadia de Montserrat, p. 327-339.

GRIMALT, Josep A., 2003, Les rondalles de mossèn Alcover, entre el realisme i l'estil abstracte, in Jaume Guiscafrè, Antoni Picornell, Actes del Congrés Internacional Antoni M. Alcover, Barcelona, Publicacions de l'Abadia de Montserrat, p. 563-579.

GUISCAFRÈ, Jaume, 1996, Una bibliografia de les edicions i les traduccions de les rondalles de mossèn Alcover, Randa, $\mathrm{n}^{\circ}$ 38, p. 151-221.

GUISCAFRÈ, Jaume, 2003, «D'aqueixs llargs bons ja no me'n record»: La transmissió oral de l'Aplec de Rondaies Mallorquines d'en Jordi d'es Racó, in Jaume Guiscafrè, Antoni Picornell (eds), Actes del 
Congrés Internacional Antoni M. Alcover, Barcelona, Publicacions de l'Abadia de Montserrat, p. 659-668.

GUISCAFRÈ, Jaume, 2007, Antoni M. Alcover, l'instituïdor del cànon rondallístic, Ooohéee, $\mathrm{n}^{\circ} 3$, p. 58-80.

GUISCAFRÈ, Jaume, 2008, El rondallari Aguiló: Transcripció, catalogació i estudi introductori, Barcelona, Publicacions de l'Abadia de Montserrat.

GUISCAFRÈ, Jaume, 2011, Els rondallistes catalans del període romàntic i la recerca d'un model de llengua literària, in Imma Creus, Maite Puig et Joan R. Veny (eds), Actes del Quinzè Col-loqui Internacional de Llengua i Literatura Catalanes, vol. III, Barcelona, Publicacions de l'Abadia de Montserrat, p. 69-80.

GUISCAFRÈ, Jaume, Picornell Antoni (eds), 2003, Actes del Congrés Internacional Antoni M. Alcover, Barcelona, Publicacions de l'Abadia de Montserrat.

JASON, Heda, 1977, Ethnopoetry: Form, Content, Function, Bonn, Linguistica Biblica.

KARLINGER, Felix, LLOMPART, Gabriel, 1977, Quatre rondalles de Mallorca, Randa, nº 6, p. 182-201.

KERBRAT-ORECCHIONI, Catherine, 2003, Les récits conversationnels ou la parole « ordinaire ", c'est tout un art, in Jean-Baptiste Martin et Nadine Decourt (éds), Littérature orale : Paroles vivantes et mouvantes, Lyon, Presses Universitaires de Lyon, p. 99-121.

LORD, Albert Bates, 1995, The Singer Resumes the Tale, ed. by Mary Louise Lord, Ithaca, Cornell University Press.

MANILA, Gabriel, 2005, Com una rondalla: Els treballs i la vida de mossèn Alcover, Manacor, Fundació Pública Antoni Maria Alcover.

MANILA, Gabriel, 2009, Pròleg, in Ballester, Ramon, 2009, Les rondalles més serverines, Palma de Mallorca, Editorial Moll, p. 13-17.

MARCH, Joan, 2001, Mossèn Alcover i el món de la ciència: La creació del llenguatge científic català modern, Palma, Lleonard Muntaner Editor.

MASSOT, Josep, 1985, Antoni M. Alcover i la llengua catalana, Barcelona, Publicacions de l'Abadia de Montserrat.

MOLL, Francesc de Borja, 2003, Obres completes I: Escrits autobiogràfics, Ed a/s de Maria Pilar Perea, Palma de Mallorca, Editorial Moll.

MOLL, Francesc de Borja, 2004, Obres completes II: Mossèn Alcover i l'Obra del Diccionari, Ed a/s de Maria Pilar Perea, Palma de Mallorca, Editorial Moll.

NАвоKоV, Vladimir, 1996, Laughter in the Dark, London, Penguin, p. 1.

NILES, John D., 1999, Homo Narrans: The Poetics and Anthropology of Oral Literature, Philadelphia, University of Pennsylvania Press.

ONG, Walter, 2012, Orality and Literacy: The technologizing of the word, New York, Routledge [1st ed. 1982].

ORPÍ, Père, 1996, Rondaies mallorquines inédites, Palma de Mallorca, Lleonard Muntaner Editor.

ZUMThOR, Paul, 1983, Introduction à la poésie orale, Paris, Éditions du Seuil. 


\section{NOTES}

1. Cet article se situe dans le cadre d'une recherche sur la littérature populaire catalane qui a reçu le soutien et le financement du ministère de l'Économie et de la Compétitivité grâce au projet $R \& D$ de "Littérature populaire catalane dans la seconde moitié du $\mathrm{Xx}^{\mathrm{e}}$ siècle: la documentation, l'étude et la diffusion» (FFI 2012-31808). Pour une première approche du personnage et de l'œuvre d'Alcover, il est indispensable de consulter MASSOT (1985), MARCH (2001), GUISCAFRÈ/PICORNELL (2003), MOLL (2004) et MANILA (2005). En ce qui concerne son œuvre lexicographique, il est recommandé de visiter le portail Alcover géré par Maria Pilar Perea, consulté le 23 mars 2015.

2. En ce qui concerne l'activité et la production de contes d'Alcover, on peut consulter GRIMALT $(1978,1996)$ et GUISCAFRÈ $(1996,2007)$.

3. Antonio DE TRUEBA Y DE LA QUINTANA (Galdames, 1919 - Bilbao, 1889) est l'auteur d'une œuvre vaste et très inégale. Ses recueils de contes de mœurs d'inspiration populaire méritent d'être signalés.

4. En 1996, à l'occasion du centenaire de la publication du premier volume de l'Aplec, commença l'édition classée et commentée par les soins de Josep A. Grimalt avec la collaboration de Jaume Guiscafrè. Six volumes ont été publiés jusqu'à maintenant, lesquels comprennent les contes d'animaux, les contes merveilleux et les contes religieux.

5. J'adhère à la distinction proposée par GRIMALT $(1996,22)$ et je la développe ici.

6. Ces deux prologues sont reproduits, refondus, dans ALCOVER $(1996,45-57)$. En ce qui concerne l'article, j'utilise et cite la réédition qu'en fit MASSOT (Alcover, 1983).

7. Giuseppe Pitrè avait déjà émis des doutes vis-à-vis de l'« authenticité » de certaines versions dans sa note sur les deux premiers volumes de la collection qu'il avait publiée en 1897 dans l'Archivio per lo studio delle tradizioni popolari. À ce sujet, voir GRIMALT (1996, 20-24).

8. Une bonne preuve de l'intérêt d'Alcover pour l'étude scientifique du folklore est que, parmi les livres qui faisaient partie de sa bibliothèque personnelle, on trouve quelques ouvrages des auteurs les plus importants de l'école historico-géographique finlandaise: Leitfaden der vergleichenden Märchenforschung (1913), Ethnische Märchen-und Sagenvarianten (1918) et les trois volumes de Vergleichende Rätselforschungen (1918-1920) d'Antti AARNE; le premier volume des Kalevalastudien (1924) de Kaarle KROHN; Die finnischen und nordischen Varianten des zweiten Merseburgerspruche (1914) et The Tale of the Two Travellers (1916) de Reidar Th. CHRISTIANSEN, ou Kaiser und Abt (1923) de Walter ANDERSON. De même, le Bolletí del Diccionari de la Llengua Catalana, fondé et dirigé par Alcover, a également publié des notes concernant d'autres ouvrages parues dans le Folklore Fellows' Communications, notamment les numéros 13 à 16, 19 et 21 de la collection. J'ignore dans quelle mesure Alcover a pu, à cette époque, assimiler ces lectures parce que, bien que j'aie pu voir ces exemplaires dans sa bibliothèque, je ne les ai pas examinés avec attention. Je ne pense pas, en tout cas, qu'il en ait retiré un grand profit. Mais s'il ne s'agit pas de donations, le simple fait qu'Alcover se soit préoccupé d'acquérir ces ouvrages et d'en faire un compte rendu montre bien sa volonté de se tenir au courant des études folkloristiques réalisées en ce premier quart du $\mathrm{Xx}^{\mathrm{e}}$ siècle par un des centres les plus importants au niveau international en matière de folklore narratif.

9. manila $(2009,16)$ fait remarquer de façon pertinente que «trois quarts du matériel, 293 contes, fut recueilli dans la région de Manacor et Son Servera et dans quatre villages seulement. À savoir, 176 à Manacor, 66 à Son Servera, 35 à Sant Llorenç des Cardassar et 16 à Son Carrió. Face à ces données, nous pouvons nous permettre d'affirmer que l'Aplec de Rondalles Mallorquines est constitué à partir de matériel recueilli dans ces quatre localités ». Alcover luimême reconnaît cette limitation $(1996,47)$. 
10. À propos de la nature des textes contenus dans ces six cahiers et leur importance pour l'étude du conte oral majorquin et du processus de création d'Alcover, voir GRIMALT (1994).

11. J'ai traité cette question plus en profondeur dans GUISCAFRÈ (2011).

12. À propos de la collection de contes d'Aguiló et de l'influence qu'il a exercée sur Alcover, voir GUISCAFRÈ (2008).

13. BALLESTER, qui a enquêté sur les narrateurs/informateurs originaires de Son Servera, signale qu'Alcover "n'était pas très minutieux dans sa prise de notes, en bas de page, de l'identité complète » et que c'est la raison pour laquelle « la tâche d'identification a été difficile et que l'on n'a pratiquement rien retrouvé de certains d'entre eux » $(2009,35)$. Il reste encore - et il est peutêtre trop tard - à faire une enquête en profondeur au sujet des narrateurs/informateurs qui fournissaient des récits à Alcover.

14. À cet égard, je ne suis pas d'accord avec NILEs $(1999,102)$ qui attribue le statut de folklore act à la "folkloric performance [...] that is commissioned and recorded by outsiders for the primary purpose of generating a record of it for their own textual communities". KERBRAT-ORECCHIONI $(2003,101)$, quant à elle, fait la distinction entre "récits conversationnels spontanés ", "récits oraux "élicites" en situation d'entretien ", " différentes formes de l'“oraliture" » et " littérature narrative écrite » : les récits des conteurs en performance appartiennent à la troisième catégorie, les textes manuscrits contenus dans les cahiers appartiennent à la deuxième et les versions publiées d'Alcover, à la dernière.

15. À propos de ces processus de reconstruction, voir GRIMALT (1998).

16. Pitrè justifie ses réserves concernant l'« authenticité » des versions d'Alcover en se fondant entre autres sur leur longueur excessive.

17. Pour une présentation argumentée et commentée avec des exemples de ces aspects, voir GUISCAFRÈ $(2007,69-76)$.

18. L'Aplec devrait avoir un statut canonique semblable à ceux de Kinder-und Hausmärchen des frères Grimm en Allemagne ou des Contes de Perrault en France. Que l'on n'ait pas encore publié d'anthologie dans la collection «Les Millors Obres de la Literatura Catalana » est quelque chose d'anormal, imputable aussi bien à certaines dynamiques socio-linguistiques et interdialectales propres à la langue catalane qu'à la myopie des canonisateurs.

19. KARLINGER $(1977,182)$ fait cette remarque : «Quand celui qui écrit ces lignes, parcourut l'île de Majorque, cela fait vingt-cinq ans, à la recherche de rondalles, il était possible de trouver, presque partout, de bons narrateurs mais, de l'analyse des narrations recueillies, il résulta que la plupart figuraient déjà dans la publication de l'archiduc Lluís Salvador ou dans la compilation du spécialiste majorquin mossèn Antoni Maria Alcover. Il est clair que certaines de ces rondalles étaient des variantes, mais en ce qui concerne le thème, la dépendance vis-à-vis des versions de mossèn Alcover était indéniable et il était difficile de savoir si l'île avait déjà été explorée de façon exhaustive ou si les textes imprimés des rondalles avaient eu une répercussion directe ou indirecte sur les habitants ». Pour ma part, j'ai déjà démontré que, effectivement, certains conteurs oraux avaient acquis un volume quelconque de l'Aplec et en avaient mémorisé ou appris des versions qu'ils racontaient plus tard devant leur auditoire habituel (Guiscafrè, 2003).

20. La maison d'édition Moll commercialise encore ces enregistrements, remastérisés, dans une collection de douze CD. 


\section{RÉSUMÉS}

C'est par intérêt personnel et en raison de son amour de la lecture qu'Antoni M. Alcover redécouvrit et remit en valeur la tradition orale des contes et des histoires de Majorque. Il recueillit, remania et reconstruisit pendant cinquante ans et de façon intermittente plus de quatre cents histoires de source orale. Alcover fut le premier folkloriste qui reconnut explicitement le travail des conteurs et informateurs qui lui avaient fourni les versions à partir desquelles, grâce à ses dons narratifs, il bâtit les textes qu'il réunit en douze volumes de l'Aplec de rondaies mallorquines d'en Jordi d'es Racó (1896-1931). Les conteurs, auditeurs et lecteurs postérieurs l'adoptèrent et ils le consacrèrent non seulement comme la référence principale et canonique en matière de récit oral populaire mais aussi comme l'unique.

Due to his personal interest in storytelling and his love of reading, Antoni M. Alcover rediscovered and reconsidered Mallorcan tales and stories from the oral tradition. He collected, reworked and rebuilt over four hundred stories from oral sources intermittently for fifty years. Alcover was the first Catalan folklorist who explicitly recognized the work of storytellers and informants who provided him with versions of oral material from which, thanks to his narrative gifts, he built the texts subsequently published in the twelve volumes of the Aplec de rondaies mallorquines d'en Jordi d'es Racó (1896-1931). Later storytellers, listeners and readers regard him not only as their main reference in terms of canonical and popular storytelling, but also as a unique figure

\section{INDEX}

Thèmes : anthropologie (Europe), littérature orale nomsmotscles Catalans

Mots-clés : conte, collecte, réécriture des contes

Keywords : Tale, Collecting, Rewriting, Majorca, Balearic Isles

Index géographique : Majorque, Baléares (îles)

\section{AUTEUR}

\section{JAUME GUISCAFRÈ}

Université des îles Baléares 